\title{
Les choix politiques des slavophones de Macédoine dans la guerre civile grecque
}

The political choices of the Slavophones in Greek Macedonia during the Greek civil war

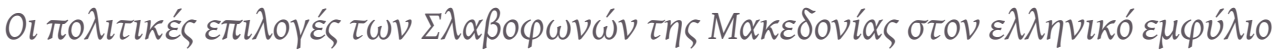
$\pi o ́ \lambda \varepsilon \mu o$

\section{Raymond Alvanos}

Traducteur : Joëlle Dalègre

\section{OpenEdition}

\section{Journals}

Édition électronique

URL : https://journals.openedition.org/ceb/1160

DOI : $10.4000 /$ ceb. 1160

ISSN : 2261-4184

Éditeur

INALCO

Édition imprimée

Date de publication : 9 janvier 2012

ISSN : 0290-7402

\section{Référence électronique}

Raymond Alvanos, «Les choix politiques des slavophones de Macédoine dans la guerre civile grecque », Cahiers balkaniques [En ligne], 40 | 2012, mis en ligne le 27 mai 2012, consulté le 06 juillet 2021. URL : http://journals.openedition.org/ceb/1160; DOI : https://doi.org/10.4000/ceb.1160

Ce document a été généré automatiquement le 6 juillet 2021

\section{cc) (†) 8}

Cahiers balkaniques est mis à disposition selon les termes de la Licence Creative Commons Attribution - Pas d'Utilisation Commerciale 4.0 International. 


\title{
Les choix politiques des slavophones de Macédoine dans la guerre civile grecque
}

\author{
The political choices of the Slavophones in Greek Macedonia during the Greek \\ civil war

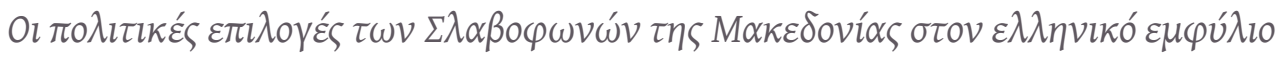 \\ $\pi \lambda^{\prime} \varepsilon \mu o$
}

\section{Raymond Alvanos}

Traduction : Joëlle Dalègre

1 Un petit nombre des habitants de la Macédoine grecque est né et a été élevé dans des familles dont la langue maternelle - ou celle de leurs grands-parents - appartenait aux langues slaves ${ }^{1}$. L'étude de leur identité politique présente un intérêt tout particulier, spécialement si l'on comprend la signification de l'identité comme une dialectique entre les questions, les besoins et les attentes des hommes d'une part, et, d'autre part, la politique de l'État, c'est-à-dire le mécanisme qui coordonne la production et la répartition des ressources, en même temps qu'il impose des lois contraignantes aux habitants d'une région géographiquement définie. Dans le cas particulier des paysans slavophones de Macédoine, les politiques de l'État grec n'ont pas été les seules à influencer leur vie. Ils se sont trouvés à l'épicentre d'une «frontière » multi-composite et multilatérale où se croisaient des politiques et des idéologies nationales contraires, où, en plus de la politique de l'État grec, les choix politiques du $\mathrm{KKE}^{2}$, de l'Italie, de la Yougoslavie et de la Bulgarie se sont révélés décisifs pour leur vie. Comme on le verra plus tard, les slavophones ne constituaient pas une seule catégorie avec des choix politiques identiques, même si, après la fin de la Seconde Guerre Mondiale, ils ont été, presque en totalité, regardés avec méfiance et parfois haine, par la quasi-totalité de la société grecque.

Les slavophones Macédoniens sont devenus l'objet d'un intérêt systématique de la part des services de l'État grec depuis la décennie des années 1920. Selon une statistique 
officielle secrète, en 1925 - donc après les échanges de populations - environ 160000 slavophones habitaient la Macédoine grecque, pour la plupart dans les régions de Florina, de Kastoria et de Pella. Dans une écrasante majorité, il s'agissait de petits agriculteurs, qui avaient bénéficié de la grande redistribution des terres de la décennie 1929, même si les revendications des réfugiés avaient réduit la part des terres qu'ils espéraient obtenir de l'héritage des Ottomans chassés. Sur le plan politique, la plupart des slavophones s'étaient identifiés au Parti Populaire et à ses députés nationalistes qui étaient d'anciens Macédonomaques ${ }^{3}$. L'implantation rurale des slavophones, leur intégration dans le système politique et clientéliste, leurs liens avec les députés les plus nationalistes (comme les antivénizélistes Tsondos Vardas et Anastasios Dalipis), la présence électorale minime du KKE et le peu de soutien de l'organisation nationaliste ORIM (Organisation révolutionnaire intérieure de Macédoine) sont des indices de leur désir d'une intégration pacifique dans l'État grec.

Cependant, la suppression en 1936 des structures parlementaires eut des conséquences importantes sur les procédures de médiation entre l'État et la population slavophone. Tandis que, dans le régime parlementaire, les Slavophones ${ }^{4}$ avaient la possibilité de se sentir participer au jeu politique par le système clientéliste et leurs liens avec les " patrons » locaux, l'arrivée de Métaxas au pouvoir mit fin à ce processus d'intégration dans la société grecque. En même temps, elle a donné le pouvoir aux partisans de l'homogénéisation nationale de la région qui, depuis l'arrivée des réfugiés déjà, soutenaient la nécessité, soit d'une hellénisation immédiate des slavophones, soit de leur expulsion du pays. L'intervention soutenue de l'État dans leur vie quotidienne en vue d'une hellénisation forcée a fait naître chez certains d'entre eux une haine qui ne s'est pas adressée simplement au nouveau gouvernement, mais aussi à l'État grec dans son ensemble.

4 Pendant l'Occupation, l'ensemble des Slavophones a été appelé à soutenir des forces opposées, les Italiens, les Allemands, le KKE, mais aussi -facteur nouveau -, les communistes yougoslaves qui répandaient l'idéologie nationale "macédonienne " et l'idée de l'Union future de la Macédoine de l'Égée et de la Macédoine yougoslave. Le KKE cependant, s'il reconnaissait l'existence d'une minorité nationale slavomacédonienne, ne lui promettait que le respect de ses droits sans changement des frontières de la Grèce. Une partie des Slavophones s'est engagée aux côtés des Italiens, une autre a intégré l'Ochrana ${ }^{5}$ et les organisations du Comité bulgaro-axo-macédonien qui étaient assez actives principalement dans les régions de Kastoria et d'Edessa. Le KKE, par la création du SNOF («EAM» en slavomacédonien) a essayé (et en grande partie réussi) à détacher les Slavophones des organisations ci-dessus qui les avaient armés et à les intégrer dans la nouvelle organisation. La plupart des Comitadjis ont rendu leurs armes sans combat, et certains ont rejoint le SNOF. D'autres ont persisté à collaborer avec les Allemands et ont pris part à des conflits armés contre l'ELAS et le SNOF, allumant la mèche d'une petite "guerre civile » à l'intérieur même de l'élément slavophone $e^{6}$.

\section{La base sociale des conflits}

Pour bien saisir la base sociale des conflits qui ont marqué la guerre civile, il est indispensable d'insister sur la situation économique désespérée de la campagne macédonienne à la fin de l'Occupation. La lutte intense pour des ressources naturelles 
largement insuffisantes offrait une base pour un antagonisme social qui, progressivement, s'est transformé en un conflit ouvert violent. Le grand problème de la période suivant la fin de l'Occupation, fut la sous-alimentation à laquelle s'ajoutaient, à cause du grand nombre de maisons brûlées pendant la guerre, un problème d'hébergement et l'insuffisance des biens élémentaires dans toute la Grèce du Nord. Dans le département de Florina, où environ 62 des 88000 habitants étaient Slavophones, il y avait 165 familles sans terres, 1110 familles avec une propriété inférieure à 10 stremmata (soit 1 hectare) et 1743 familles avec une propriété entre 11 et 25 stremmata (1,1 à 2,5 hectares). En d'autres termes, 3018 familles, presque $40 \%$ des ruraux qui formaient l'écrasante majorité de la population du département, possédaient des terres inférieures au minimum viable de 25 stremmata $^{7}$.

Cette pression était encore plus forte dans le nome de Kastoria où le nombre des Slavophones était évalué à $42 \%$ de la population, environ 27000 personnes au total, dont la majorité habitaient des villages au nord et à l'est de la ville de Kastoria. Selon les données du chef du Service de l'agriculture de ce département, Vassilis Ioannou, un quart des habitants possédait une terre de moins d'un hectare caractérisée comme «insuffisante » et $52 \%$ de la population disposaient d'une "toute petite propriété " inférieure au seuil de survie, entre 1 et 3 hectares. Au total, $77 \%$ de la population du département possédaient des surfaces inférieures au seuil de survie de 3 hectares ${ }^{8}$. L'offre pléthorique de main-d'œuvre agricole dans le département créa un vaste bassin d'actifs sans emploi qui, dans la situation politique explosive des années 1945-1946, produit un mélange particulièrement volatile qui agit comme catalyseur et déclencha la violence. La dynamique sociale de conflit venue " de l'intérieur », déjà apparue pendant l'Occupation, allait rencontrer le choc international venu "de l'extérieur ", né de la Guerre Froide, et ainsi alimenter une guerre civile où des milliers d'hommes seraient prêts à tuer ou à être tués.

\section{La vague des répressions contre les slavophones et ses conséquences}

7 Dans ce contexte social où dominait le manque des moyens de survie élémentaire, il n'est pas étonnant que tous ceux qui avaient durement souffert des forces d'Occupation, aient tourné leurs armes contre les slavophones. En 1945, les Allemands étaient déjà loin de la Grèce; mais leurs collaborateurs, les paysans slavophones qui avaient rejoint les organisations philobulgares, l'Ochrana et le Comité philo-axobulgare, qui avaient été armés contre l'ELAS par les Italiens, puis par les Allemands, étaient là! Les ex-comitadjis, même si le plus souvent ils n'étaient pas responsables directement des crimes des forces d'occupation, ont payé en bloc, pour tous, et se sont retrouvés après Varkiza, sans défense, à la merci des groupes armés illégaux, alors même que la plupart des responsables directs d'actes criminels, avaient déjà été châtiés par l'ELAS ou s'étaient réfugiés en Yougoslavie. La violence ne s'est pas tournée seulement contre les ex-comitadjis, mais contre tous les Slavophones, sous la forme, le plus souvent, de la mise à sac de leurs villages par les paysans armés qui avaient souffert de l'Occupation. Outre ces raids de groupes armés, les slavophones eurent à faire face également aux pressions de l'État, arrestations et poursuites judiciaires. Dans les départements de Kastoria et de Pella, ils furent surtout accusés de collaboration 
avec les Allemands, dans celui de Florina, où le Comité n'avait pas été très actif, les accusations portaient surtout sur une action en faveur de l'autonomie de la région.

Mais, peut-être encore plus douloureux que les attaques armées et les poursuites judiciaires, fut le blocus, l'impossibilité d'avoir accès aux aides de l'UNRRA ${ }^{9}$, facteur vital pour l'époque. Selon un rapport du consul britannique à Salonique, Peck, pendant toute l'année 1945, les slavophones ont souffert de ségrégation dans la distribution de l'aide; selon lui, les responsables étaient principalement les officiers grecs de rang inférieur qui, globalement, voyaient tous les slavophones comme des collaborateurs du Comité bulgare ${ }^{10}$. Il faut noter le point de vue des historiens de la Macédoine exyougoslave sur ce sujet. Selon Risto Kyrjazovski «La persécution contre les Macédoniens eut toutes les caractéristiques d'un génocide... Toutes les mesures des autorités grecques visaient à anéantir la population macédonienne ou à la contraindre à l'exil $"^{11}$. Des slavophones locaux qui ont vécu la guerre civile partagent ce point de vue sur l'existence d'un plan organisé pour leur disparition, même si plus tard, ils se sont identifiés à la conscience nationale grecque. Selon le témoignage de l'un d'eux, originaire d'un village de Kastoria "Ils pensaient qu'ils allaient nous anéantir, comme les Turcs avec les Arméniens, que nous allions partir et ne jamais revenir ».

Certains historiens grecs, comme Ioannis Koliopoulos attestent de cette vague de persécutions, même si ce dernier ne parle pas de "génocide", mais du "manque de soins injustifié et inhumain » dont le gouvernement et ses agents ont fait preuve face aux slavophones et écrit : « des bandes armées de gens de droite, principalement des réfugiés, détruisaient et immolaient sans opposition et souvent même, avec la tolérance des autorités $»^{12}$.

10 Les persécutions vécues par les slavophones après les accords de Varkiza eurent avant tout un caractère ethnique et, secondairement, un caractère politique. Dans la région de Yannitsa, un ami écrivait à Dragoumis en mai 1945 "Les bulgarophones sont quasiment en état de persécution [...] Mais le malheur, c'est que la persécution se tourne sans distinction aussi bien contre les Macédonomaques et tout Grec bulgarophone, même animé de sentiments pro-grecs très forts et qui a à son actif un passé patriote. Malheureusement, ce qui domine, c'est le « Tout ce qui n'est pas Grec est barbare », ou ici « Tout bulgarophone est Bulgare $»^{13}$.

11 Les discriminations dans la répartition des aides de l'UNRRA, les poursuites judiciaires et les razzias des paysans armés, rendirent très difficile aux paysans slavophones la survie sur leurs terres. La fuite de milliers d'entre eux loin de leurs villages, soit vers la montagne, soit en Yougoslavie, est due à une peur omniprésente. On estime qu'entre 1944 et 1946, 10000 slavophones, principalement de Macédoine orientale, se sont réfugiés en Bulgarie, et 17000 en Yougoslavie, dont 13000 en raison des poursuites après Varkiza ${ }^{14}$. Ils y furent considérés comme des frères irrédimés, on leur fournit des soins mais aussi les «lumières" aptes à leur faire "prendre conscience " de leur «essence nationale» macédonienne. Avec les autres Grecs pourchassés qui se réfugièrent en Yougoslavie, ils allaient constituer le noyau de l'Armée Démocratique dans la guerre civile qui commençait. Comme l'écrivait avec beaucoup de clairvoyance, en 1948, Dimitrios Zaphiropoulos, un ancien militaire qui étudia la guerre civile, « La fuite massive en 1945 de membres de l'ELAS et d'autonomistes vers la Serbie n'a pas été évaluée comme il l'aurait fallu par les autorités locales qui s'en réjouissaient et imaginaient qu'en ne réagissant pas, elles seraient ainsi débarrassées de cet élément encombrant ... » [...] «C'est seulement en 1946, avec le retour de Yannoulis ${ }^{15}$, qu'ils 
comprirent leur erreur, virent qu'une révolution se préparait et comprirent qu'au lieu d'encourager les départs, il fallait prendre des mesures pour les prévenir et les contenir ${ }^{16} »$.

\section{Les élections de 1946}

12 À première vue, l'abstention aux élections de 1946 demandée par les partis de gauche ne semble pas avoir eu des conséquences importantes sur les résultats électoraux de Macédoine Occidentale. Cependant, une analyse en profondeur portant uniquement sur les régions slavophones fait apparaître une image différente, surtout si on la met en relation avec la composition de la population de chaque village.

Dans le tableau ci-dessous, on a choisi les centres où votaient des électeurs de villages uniquement slavophones, pour étudier ainsi les comportements électoraux de ces paysans.

14 Le tableau montre que les paysans slavophones qui ont voté aux élections de mars 1946 dans le nome de Florina représentaient environ $7,5 \%$ de la population. Comme sur la base des données d'avant-guerre, le pourcentage escompté de participation au vote est d'environ $20 \%$ des inscrits, il en ressort qu'environ $60 \%$ des slavophones de la région de Florina se sont abstenus, alors qu'en Grèce le pourcentage d'abstention est évalué à $25 \%^{17}$.

1. Résultats électoraux et participation dans les circonscriptions du département de Florina où ne votaient que des slavophones, le 31/3/46. (IPE ${ }^{18}$ )

\begin{tabular}{|l|l|l|l|l|l|l|}
\hline VILLAGES & $\begin{array}{l}\text { Popu } \\
\text { lation }\end{array}$ & Votants & $\begin{array}{l}\text { \%otants/ } \\
\text { Population }\end{array}$ & IPE \% & EPE \% & KF \% \\
\hline Véis & 2450 & 66 & $2,69 \%$ & $34,85 \%$ & $30,30 \%$ & $34,85 \%$ \\
\hline Achlada (Skopos) & 2388 & 188 & $7,87 \%$ & $19,15 \%$ & $32,45 \%$ & $27,66 \%$ \\
\hline $\begin{array}{l}\text { Polypotamos } \\
\text { (Atrapos,Triandafyllia }\end{array}$ & 2601 & 31 & $1,19 \%$ & $19,35 \%$ & $61,29 \%$ & $0,00 \%$ \\
\hline Boufios & 1989 & 33 & $1,66 \%$ & $21,21 \%$ & $12,12 \%$ & $57,58 \%$ \\
\hline Aghios Panteleimon & 1747 & 251 & $14,37 \%$ & $34,26 \%$ & $11,95 \%$ & $52,59 \%$ \\
\hline Aetos & 1189 & 67 & $5,63 \%$ & $13,43 \%$ & $62,69 \%$ & $14,93 \%$ \\
\hline Xyno Nero & 1955 & 49 & $2,51 \%$ & $67,35 \%$ & $12,24 \%$ & $18,37 \%$ \\
\hline $\begin{array}{l}\text { Pyxo (Agh.Achillios, Psarades, } \\
\text { Vrondero) }\end{array}$ & 2232 & 362 & $16,22 \%$ & $68,63 \%$ & $9,80 \%$ & $21,57 \%$ \\
\hline $\begin{array}{l}\text { Andartikou (Trigono, Sfika, } \\
\text { Tyrnovo) }\end{array}$ & 2497 & 169 & $6,77 \%$ & $14,09 \%$ & $35,64 \%$ & $37,85 \%$ \\
\hline Vatochoriou (Krystallopigi,Kottas) & 1920 & 51 & $2,66 \%$ & $41,42 \%$ & $39,05 \%$ & $15,38 \%$ \\
\hline
\end{tabular}




\begin{tabular}{|l|l|l|l|l|l|l|}
\hline Kellis & 1577 & 238 & $15,09 \%$ & $0,00 \%$ & $57,98 \%$ & $41,18 \%$ \\
\hline Sitaria (Palesta, Lofoi) & 1913 & 238 & $12,44 \%$ & $28,99 \%$ & $17,23 \%$ & $44,12 \%$ \\
\hline Krateros (Ethniko, Parori) & 1781 & 256 & $14,37 \%$ & $60,55 \%$ & $20,31 \%$ & $9,38 \%$ \\
\hline Ensemble des villages & 26239 & 1999 & $7,62 \%$ & $29,01 \%$ & $30,67 \%$ & $32,32 \%$ \\
\hline Total du nome & 87931 & 8894 & $10,11 \%$ & $32,84 \%$ & $31,22 \%$ & $30,50 \%$ \\
\hline
\end{tabular}

Mais les résultats de la région de Kastoria sont encore plus révélateurs ${ }^{19}$.

2. Résultats électoraux et participation dans les circonscriptions du département de Kastoria où ne votaient que des slavophones, le 31/3/46.

\begin{tabular}{|l|l|l|l|l|l|l|}
\hline Villages & Population & Votants & \%Votants/Pop. & IPE \% & EPE \% & KF \% \\
\hline Agh. Dimitrios & 223 & 21 & $9,42 \%$ & $14,29 \%$ & $42,86 \%$ & $42,86 \%$ \\
\hline Vapsorio & 820 & 15 & $1,83 \%$ & $60,00 \%$ & $26,67 \%$ & $6,67 \%$ \\
\hline Vyssinia & 664 & 9 & $1,36 \%$ & $66,67 \%$ & $33,33 \%$ & $0,00 \%$ \\
\hline Gavro & 355 & 7 & $1,97 \%$ & $57,14 \%$ & $42,86 \%$ & $0,00 \%$ \\
\hline Dendrochorio & 800 & 14 & $1,75 \%$ & $50,00 \%$ & $42,86 \%$ & $7,14 \%$ \\
\hline Ieropigi & 458 & 27 & $5,90 \%$ & $22,22 \%$ & $3,70 \%$ & $74,07 \%$ \\
\hline Kastanophyto & 482 & 45 & $9,34 \%$ & $48,89 \%$ & $20,00 \%$ & $31,11 \%$ \\
\hline Kondorropi & 341 & 17 & $4,99 \%$ & $82,35 \%$ & $0,00 \%$ & $17,65 \%$ \\
\hline Kranionas & 623 & 27 & $4,33 \%$ & $70,37 \%$ & $3,70 \%$ & $25,93 \%$ \\
\hline Lakkomaton & 893 & 80 & $8,96 \%$ & $55,00 \%$ & $20,00 \%$ & $25,00 \%$ \\
\hline Lavki & 732 & 5 & $0,68 \%$ & $60,00 \%$ & $0,00 \%$ & $40,00 \%$ \\
\hline Makrochorio & 1031 & 25 & $2,42 \%$ & $48,00 \%$ & $16,00 \%$ & $36,00 \%$ \\
\hline Mavrokambos & 350 & 14 & $4,00 \%$ & $42,86 \%$ & $28,57 \%$ & $28,57 \%$ \\
\hline Mela & 717 & 1 & $0,14 \%$ & $100,00 \%$ & $0,00 \%$ & $0,00 \%$ \\
\hline Melissotopos & 723 & 30 & $4,15 \%$ & $30,00 \%$ & $63,33 \%$ & $6,67 \%$ \\
\hline Moschochorio & 448 & 11 & $2,46 \%$ & $9,09 \%$ & $18,18 \%$ & $72,73 \%$ \\
\hline Oxya & 313 & 525 & $16,61 \%$ & $13,46 \%$ & $1,92 \%$ & $84,62 \%$ \\
\hline Petropoulakio $\%$ & $0,42 \%$ & $0,00 \%$ & $17,65 \%$ \\
\hline
\end{tabular}




\begin{tabular}{|l|l|l|l|l|l|l|}
\hline Polyanemos & 273 & 12 & $4,40 \%$ & $75,00 \%$ & $8,33 \%$ & $16,67 \%$ \\
\hline Polykeraso & 452 & 13 & $2,88 \%$ & $61,54 \%$ & $0,00 \%$ & $38,46 \%$ \\
\hline Siderochorio & 550 & 0 & $0,00 \%$ & $0,00 \%$ & $0,00 \%$ & $0,00 \%$ \\
\hline Spilaio & 256 & 17 & $6,64 \%$ & $23,53 \%$ & $29,41 \%$ & $47,06 \%$ \\
\hline Teicheio & 869 & 42 & $4,83 \%$ & $66,67 \%$ & $16,67 \%$ & $16,67 \%$ \\
\hline Phtelia & 849 & 39 & $4,59 \%$ & $43,59 \%$ & $17,95 \%$ & $38,46 \%$ \\
\hline Chalaron & 723 & 7 & $0,97 \%$ & $57,14 \%$ & $42,86 \%$ & $0,00 \%$ \\
\hline TOTAL & 14210 & 547 & $3,85 \%$ & $46,98 \%$ & $19,20 \%$ & $33,64 \%$ \\
\hline
\end{tabular}

On voit sur le tableau ci-dessus que les paysans slavophones ${ }^{20}$ qui ont voté en mars 1946 ne représentaient que 3,85\% de la population (547 votants sur 14210 habitants de villages uniquement slavophones). Comme le pourcentage attendu, selon les données d'avant-guerre, est d'environ $20 \%$, il s'ensuit que près de $80 \%$ (et peut-être plus) des paysans slavophones se sont abstenus!

La forte abstention des slavophones n'était pas due uniquement à un choix conscient. Comme déjà indiqué, nombre d'entre eux étaient absents le jour des élections parce qu'ils avaient trouvé refuge en Yougoslavie ou se trouvaient en prison ou cachés dans la montagne. Malgré cela, le pourcentage d'abstentions n'en reste pas moins impressionnant. Il s'explique plus facilement si on le met en rapport avec les poursuites sévères qu'ont connues les populations de Florina et particulièrement celles de Kastoria.

17 Nombreux slavophones qui choisirent par la suite d'intégrer le camp gouvernemental se sont abstenus volontairement aux élections de 1946. Selon un habitant d'un village " mixte » de Kastoria qui servit dans l'armée gouvernementale et se définissait luimême comme «de Droite ", presque personne parmi les «locaux» (= les slavophones) n'alla voter, beaucoup prétextant des travaux agricoles et s'abstenant, non pas en raison de leur identification avec le $\mathrm{KKE}^{21}$, mais pour montrer leur opposition à un État qui , selon eux, ne préservait, pensaient-ils, ni leur vie, ni leurs biens, ce qui était supposé être sa mission. Leur abstention était un message de protestation adressé au gouvernement qui, non seulement ne pouvait ou ne voulait pas les protéger, mais qui, de plus, se tournait contre eux d'une certaine façon, par les décisions judiciaires prises à leur encontre.

18 L'impression d'être exclu par l'État et la société grecque a intensifié entre 1945 et 1946 le fait, pour les paysans slavophones, d'avoir perdu les relations privilégiées qu'ils avaient eues, dans l'entre-deux-guerres, avec leurs "patrons» politiques. Dans la région de Kastoria, l'alliance, à l'intérieur de l'IPE, Mouvement Uni des Nationalistes, $\mathrm{du}$ "père » politique de nombreux slavophones dans l'entre-deux-guerres, Philippe Dragoumis, et de Périclès Iliadis, l'instituteur réfugié, - expression de la ligne nationaliste la plus dure en faveur d'un nettoyage ethnique immédiat de la région - les coupa de leurs protecteurs politiques de la période précédente. L'adjonction d'Iliadis et du courant réfugié qu'il représentait rendit possible la réconciliation des principaux 
groupes sociaux adversaires de l'Entre-deux-Guerres - indigènes et réfugiés - qui se traduisait dans l'affrontement entre Populistes et Libéraux.

La situation nouvelle créa un nouveau front «national », dirigé principalement contre les slavophones indigènes de la région, qui s'exprima dans l'IPE et obtint de grands succès dans les villages de réfugiés et de Grecs indigènes avec plus de $80 \%$ des voix, au moment où il avait 55,12 \% des voix dans le reste de la Grèce.

Le département de Kastoria a accordé à la coalition anticommuniste $25 \%$ de voix en plus que le reste de la Grèce, inaugurant ainsi une identification politique à la Droite qui allait se poursuivre pendant de nombreuses décennies. Déjà pendant l'Occupation, les efforts du KKE pour réconcilier les Grecs et les Slavomacédoniens (comme il appelait les slavophones) avaient été jugés ridicules par de nombreux Grecs et avaient renforcé les rumeurs selon lesquelles le KKE menait une "politique de trahison" qui visait à céder une part de la Macédoine à la Yougoslavie. La très forte insécurité que ressentaient les hellénophones indigènes et les réfugiés - dont l'expérience de l'exil était récente - devant la perspective d'une domination des communistes et d'une possible intégration - souvent brandie devant eux - de la région à la Yougoslavie ont été les raisons principales qui les ont poussés par l'extrême droite du camp anticommuniste.

21 La très forte et impressionnante abstention des slavophones lors des élections de 1946 dans la région de Kastoria ne signifie que tous avaient continuellement un comportement politique semblable. Comme le montrèrent les résultats électoraux de Florina, ils étaient divisés entre leur loyalisme traditionnel et l'occasion qui se présentait de revendiquer une identité ethnique et politique.

\section{La fondation et l'action du NOF}

C'est le NOF (Front populaire-national de Libération, en langue macédonienne), fondé en avril 1945 à Skopje, qui entreprit de communiquer aux slavophones macédoniens de Grèce l'identité nationale du "Macédonien ", liée à l'identité politique communiste. Sa base était composée de communistes slavophones du groupe Gotse, anciens membres du KKE, qui s'étaient réfugiés en Yougoslavie, chassés par l'ELAS en octobre 1944, pour avoir refusé d'obéir à l'ordre de transfert vers des régions plus au sud, mettant fin ainsi à leur propagande, dans la région de Florina-Kastoria, en faveur de l'autonomie de la Macédoine. Les communistes (slavo)macédoniens du NOF considérèrent les accords de Varkiza comme une trahison de leurs demandes spécifiques par le KKE, et les deux organisations ont eu des relations jusqu'au début de 1946, quand le KKE se tourna progressivement vers la lutte armée $e^{22}$.

Le programme du NOF comprenait la lutte du " peuple macédonien " pour l'union entre les «Macédoniens » et les trois morceaux de la Macédoine, en particulier la Macédoine yougoslave qu'ils voyaient jouer le rôle du Piémont dans l'unité italienne. Le NOF visait à insuffler l'identité nationale "macédonienne" aux paysans slavophones de la Macédoine grecque et à répandre la perspective d'une union de la région à la république populaire de Macédoine dans le cadre de la Yougoslavie. Selon le chercheur Evangelos Kofos, le NOF fonctionnait comme une section du Parti Communiste de "Macédoine " pour la "Macédoine de l'Égée " (c'est-à-dire grecque) ${ }^{23}$. D’après des sources du NOF, il semble qu'il n'ait pas eu beaucoup de soutien dans la population locale jusqu'en novembre 1945, ce qui changea peu avant les élections de 1946, quand il 
vit son rayonnement augmenter de manière importante dans la région de Pella où il compta 7014 membres $^{24}$. Il faut noter que, dans ses proclamations aux habitants des départements de Kastoria et Pella en janvier 1946, le NOF dément la «propagande de la réaction» qui le dénonce comme une organisation autonomiste, et insiste sur le fait qu'il est la continuation de l'ELAS, qu'il est antifasciste et organise la minorité «macédonienne" de Grèce pour obtenir l'égalité en droits à l'intérieur des frontières grecques $^{25}$. Par ailleurs, comme le montrent les textes de l'organisation, elle s'efforça de recruter aussi des Grecs "démocrates", même réfugiés, opposés aux accords de Varkiza, ce qu'elle n'aurait pu faire si ses proclamations avaient parlé clairement d'annexion de la région à la République Populaire de Macédoine ${ }^{26}$.

Nous ne pouvons être certains des intentions véritables des cadres du NOF mais le fait que commencèrent alors les efforts pour une entente entre le NOF et le KKE, est vraisemblablement à relier à ces proclamations du NOF sur l'égalité en droits de la minorité «macédonienne » à l'intérieur de l'État grec ${ }^{27}$. Par ailleurs, comme on le voit dans d'autres exposés de l'organisation, il y eut un effort pour enrôler également des Grecs « démocrates ", même des réfugiés, qui étaient opposés aux accords de Varkiza, ce qui ne pouvait être atteint si les proclamations avaient parlé clairement d'une possible annexion de la région à la république de Macédoine de Yougoslavie ${ }^{28}$.

En novembre 1946, le NOF a fusionné ses groupes avec l'Armée Démocratique (ou A.D) en échange du respect de sa culture, de l'égalité en droits avec les autres Grecs et de sa représentation parmi les cadres de l'A.D. Dans la guerre civile qui commençait, le KKE désirait clairement "nationaliser » la minorité slavophone et la gagner à ses côtés. De manière caractéristique, le journal de l'Armée Démocratique, «Exormisi " (La Ruée, L'Assaut) de janvier 1948 rapporte que fonctionnent 35 écoles «slavomacédoniennes " et insiste parallèlement sur le fait que « les Slavomacédoniens comprennent bien qu'ils ne doivent ces conquêtes «nationales » qu'au combat de l'AD. C'est pourquoi ils voient cette lutte comme la leur, et la renforcent de toutes les manières $»^{29}$. Dans un autre numéro du journal, un mois plus tard, sous le titre « le premier congrès du front de libération national populaire des Slavomacédoniens NOF », on rapporte les discours des cadres, Striggos et Keramitsis, qui donnent de nombreux renseignements sur les poursuites contre les Slavomacédoniens. Le sous-entendu qui traverse la presse de l'A.D est clair: les Slavomacédoniens n'ont pas d'autre solution que de rejoindre le KKE puisque l'État grec les pourchasse avec fureur ${ }^{30}$.

\section{L'Armée Démocratique, unique voie}

Et réellement, pour une partie des slavophones, l'engagement dans l'A.D semblait l'unique voie possible. Les attaques de la Droite armée, les poursuites judiciaires, les arrestations et le blocus économique les poussaient à se placer aux côtés du KKE. Les témoignages oraux de la région de Kastoria sont très nombreux (plus de 50), et tous remarquablement concordants, à décrire comment beaucoup de slavophones ont tenté de quitter la ville pour ne plus vivre dans cette situation de guerre et éviter d'être enrôlés par les andartès ("résistants " car ils se considèrent comme la $2^{\mathrm{e}}$ résistance contre le fascisme) de l'A.D. Mais la plupart ont été rossés par les gendarmes et les hommes des MAY ou des $\mathrm{MAD}^{31}$ et contraints à rentrer dans leurs villages où ils subissaient les pratiques d'enrôlement de l'A.D et se retrouvaient, eux aussi (et, indirectement ou directement, leurs familles) à ses côtés. 
ne peut qu'être impressionné par l'unanimité des témoignages à propos du rôle du maréchal des logis Liakopoulos qui dirigeait un détachement de gendarmerie: tous s'accordent sur le fait qu'il était un " agent payé par les communistes » pour rosser les slavophones et les pousser ainsi vers la montagne. D'après ces témoignages, « souvent, les communistes dressaient une embuscade aux gendarmes, mais s'ils voyaient Liakopoulos parmi eux, ils ne frappaient pas, car ils ne voulaient pas perdre leur meilleur 'agent recruteur' $»^{32}$.

Ce sentiment de mise à l'écart et de persécution par l'État grec s'inscrit de façon caractéristique dans le témoignage d'un ancien officier de renseignements de l'A.D qui, après son arrestation, a collaboré avec les enquêteurs : «Le tiers de l'Armée de bandits ( = A.D), et principalement dans les régions du Nord, est composé de Slavomacédoniens. Ils ont un meilleur moral et sont persuadés que la seule issue qui leur reste est la guerre. Aussi combattent-ils avec fanatisme. La propagande adéquate de Zachariadis les a inclus et ils croient que tout Slavomacédonien qui tombe dans les mains de l'Armée Nationale est considéré comme un Bulgare et exécuté. Ils pensent aussi qu'ils connaîtront le même sort s'ils se rendent car, pour les Salvomacédoniens, ni indulgence, ni amnistie ne sont jamais venus de l'Armée Nationale. Ils croient également que le programme du gouvernement est d'exterminer tout élément Slavomacédonien $»^{33}$.

\section{Les « Réfugiés » ${ }^{34}$}

Comme on le sait, de nombreux villages dans toute la Grèce ont été vidés, par l'armée gouvernementale, de leurs habitants poussés de force vers les grandes villes mieux contrôlées, de sorte que l'A.D. soit privée de source de ravitaillement et de recrues. Dans les régions de population slavophone, comme le nome de Kastoria, cette pratique fut plus sélective et les villages évacués comportaient exclusivement une population réfugiée ou hellénophone indigène.

Caractéristique est ainsi le cas du village de réfugiés d'Aghios Andonios du Vitsi, en octobre 1947 : il a été évacué alors qu'il se trouvait au milieu de villages slavophones qui, selon la logique militaire, auraient dû être évacués et... ne l'ont pas été. Outre Aghios Andonios, ( $L a$ Voix de Kastoria, 19/10/47 et 17/6/51) d'autres villages de montagne ont été évacués comme Kotyli, village valacophone, Lagga, hellénophone, et les villages de réfugiés de Komninades et Chionato (La Voix de Kastoria, 26/10/47) ${ }^{35}$. La raison de leur évacuation forcée était que l'armée gouvernementale ne pouvait pas garantir la sécurité des « habitants nationalistes » de ces villages.

Dans l'année 1947 l'écrasante majorité des réfugiés des villages de Kastoria, ou de tous ceux qui étaient identifiés avec l'État ou avaient des raisons de craindre les andartès, abandonnèrent la campagne peu sûre, et se réfugièrent à Kastoria et dans les grands villages contrôlés par l'armée. Presque tous les villages de réfugiés de la région de Kastoria se vidèrent de leurs habitants qui partirent vers les villes où, en plus de la protection face aux combats, ils trouvaient la nourriture assurée grâce à la riche aide de l'UNRRA. Comme le remarque très justement un exposé britannique de l'époque, "pour la première fois les paysans de la région profitèrent d'une nourriture, au lieu qu'ils la donnent, eux, d'une nourriture étrangère à leur goût et parfois repoussante, mais d'une nourriture donnée gratuitement $»^{36}$. 

lier les cadres du MAY, s'identifiaient à la ligne nationaliste la plus dure, selon laquelle la situation donnait l'occasion de chasser de Grèce tous les slavophones pour que ne restent que des "Grecs authentiques " ${ }^{37}$. C'est ce que montre la déportation obligatoire sans précédent des paysans par l'armée gouvernementale, non pas vers les villes, mais vers des secteurs contrôlés par l'A.D. Ce fut le cas en 1947, quand, selon Philippe Dragoumis, les autorités militaires de Kastoria et de Florina commencèrent à expulser des régions frontalières, par la force, les familles slavophones "vers les régions occupées par les bandits ", mesure que Dragoumis condamne, mais qui montre que la politique de nettoyage ethnique était bien dans l'esprit des dirigeants militaires locaux ${ }^{38}$. Parallèlement de nombreux rapports et exposés d'officiers grecs de l'époque insistaient sur le fait que les «nationalistes » affluaient vers les villes tandis que restait dans les villages la "population ennemie", ce qui constituait un problème pour l'armée, vu " qu'en raison de l'abandon de la campagne par les éléments nationalistes » il n'y avait plus de réseau de renseignements efficace sur les mouvements des andartès ${ }^{39}$.

Par voie de conséquence, les villages slavophones du Vitsi, dans les régions montagneuses entre Kastoria et Florina "gardèrent» leur population pendant la guerre civile, essentiellement parce qu'elle n'était pas bien accueillie dans les centres urbains bien gardés par les autorités. Dans les villages slavophones sous contrôle de l'A.D, selon un exposé du secrétaire du NOF au $2^{\mathrm{e}}$ congrès de l'organisation, dans l'hiver 1947-1948, 87 écoles "slavomacédoniennes ", avec 10000 élèves ${ }^{40}$, ont fonctionné. Même vraisemblablement exagéré, ce chiffre montre que les villages slavophones étaient "vivants " pendant la guerre civile; en revanche, les villages, à l'origine hellénophones, du Grammos, au sud de Kastoria (entre Épire et Macédoine, région où l'on comptait 150 villages environ) formaient un « désert $»^{41}$, puisque leurs habitants 
ont eu la possibilité de profiter à la fois de la sécurité des centres urbains et des distributions gratuites d'alimentation.

\title{
Les slavophones dans la guerre civile
}

Le fait que la politique de l'État grec ait poussé les slavophones dans les bras de l'A.D, et, en conséquence, à une probable adoption progressive de l'identité nationale macédonienne, ne signifie pas qu'ils aient finalement rejoint les communistes. Au contraire, une partie importante d'entre eux a suivi la politique traditionnelle conservatrice des paysans slavophones et a choisi de suivre le camp le plus sûr, le plus fort, celui de l'État en place. Il s'agit de slavophones qui réussirent à valoriser des réseaux familiaux ou politiques d'avant-guerre, en particulier les grécomans (les slavophones hellénophones) et à obtenir de vivre en ville ou d'intégrer les MAY ou l'armée grecque. Le témoignage de Mani Yorof, né en 1930 dans une famille relativement pauvre de Vasiliada (Kastoria) montre que certains slavophones ont eu une relative possibilité de choix. Son père, pendant l'Occupation, a été poussé par les autres comitadjis du village a accepté de prendre les armes "pour garder le village». Bien que n'ayant eu aucune activité de comitadji, il fut condamné après la guerre à plusieurs années de prison. En 1947, est venu d'Oxya, un cousin qui était chez les MAY et entreprit de la convaincre de la suivre :

\begin{abstract}
«Et lui, il est venu avec les gens des MAY. Il est venu et moi, je me suis caché. Moi, je suis parti et je me suis caché de tous. Ma grande soeur arrive, elle me crie, me donne un signal. Nikos est venu, me dit-elle, viens, n'aies pas peur. Moi, je suis sorti, je suis allé à la maison. Il a commencé, il me dit, écoute : ils ne font rien. Tout d'abord, ils n'ont pas d'État. Moi, je lui dis : eh, Niko, d'accord, je crois ce que tu me dis, il se peut qu'il gagnent, il se peut qu'ils ne fassent rien. Ils portent des tsarouchia (chaussons paysans de cuir, à pompons, NdT). C'est ça, un État? D'accord, ils sont habillés, c'est une armée régulière ? Entretemps comment y aller? (dans l'EES, armée nationaliste) Toi, ton père, tu le connais. Il n'aurait pas tué une mouche. On lui donné 12 ans. Il me dit: allez, viens, on va te prendre. Tu iras à Kastoria. Ils vont t'inscrire et là ils te donneront de l'aide. Tu habiteras chez ma mère. Tu porteras un peu de bois pour le poêle, tu ne feras rien d'autre. Moi, je n'y suis pas allé ».
\end{abstract}

Le refus du jeune Yorof, (17 ans à cette époque) d'aller en ville, le mit pratiquement à la disposition des andartès qui l'enrôlèrent quelques mois plus tard.

Contrairement à Yorof, de nombreux slavophones se sont enrôlés dans les Unités de sécurité, MAY ou les Unités et Détachements de Poursuite, les MAD. Selon une première liste dans le nome de Florina, sur les 925 hommes armés venus de 45 villages inscrits au printemps 1947 dans un exposé de l'A.D à l'ONU, 258 habitaient 18 villages slavophones et dans la région de Kastoria, sur un ensemble de 47 villages armées et 536 hommes des groupes paramilitaires, 13 villages et 78 hommes étaient slavophones ${ }^{42}$.

Aux gens des MAY, il faut ajouter un nombre, indéfini mais assurément important, de slavophones enrôlés dans l'armée gouvernementale. Leurs familles se sont souvent réfugiées dans les villes où elles trouvaient protection dans les structures de l'État, alors que celles qui restaient au village devaient affronter la méfiance ou/et la haine des andartès.

Très utile pour comprendre les orientations politiques de la population slavophone est l'introduction de Michalis Keramitsis (ou Keramitziev) secrétaire du NOF, en mai 1947, 
c'est-à-dire au début de la guerre civile, lors d'une réunion du NOF sur le Kaïmaktsalan, selon laquelle $10 \%$ des «Slavomacédoniens » (visiblement il utilisa le terme officiel du KKE « Slavomacédonien » et non « Macédoniens » parce que des cadres du KKE étaient présents) étaient d'anciens probulgares («partisans de Kaltsev) ou des grécomans qui, par intérêt personnel, s'étaient rangés aux côtés de la "réaction", $20 \%$ des slavomacédoniens n'avaient pris aucune part active au combat car ils étaient «froussards » ou "pacifistes sans raison », $30 \%$ avaient des sympathies pour le NOF et le peuple grec mais n'étaient pas révolutionnaires car ils ne supportaient pas les dures épreuves et craignaient pour le sort de leur famille. Selon Keramitsis, $40 \%$ formaient donc l'avant-garde combattante du peuple "slavomacédonien" qui, même s'ils ne participaient pas tous au combat, étaient prêts à s'y soumettre ». Il faut remarquer que parmi eux, à peine $3 \%$ étaient des ouvriers alors que $37 \%$ étaient des paysans. Il nous faut considérer avec prudence ces estimations de Keramitsis (en particulier son affirmation que $85 \%$ du peuple «slavomacédonien » de Grèce avaient une identité ethnique claire et se croyait Slavomacédoniens), étant donné que son introduction avait pour but de persuader les cadres présents du KKE d'accepter que les villages slavophones fassent partie du NOF exclusivement ${ }^{43}$.

Quant à l'estimation de Keramitsis, selon laquelle $40 \%$ des slavomacédoniens composerait «l'avant-garde combattante » et donc soutiendrait l'A.D, elle correspond aux estimations des services de sécurité grecs. En l'été 1947 les dirigeants politiques du pays discutaient comme d'une expulsion éventuelle des slavophones et, dans un rapport secret, le ministère des Affaires étrangères proposait l'expulsion du nome de Kastoria de 12000 slavophones sur un total de 27000 , non bien sûr en tant que «Macédoniens » comme aurait dit Keramitsis, mais comme « sympathisants bulgares ». Les chiffres étaient à peu près les mêmes pour le nome d'Edessa, alors que, sur un total d'environ 62000 slavophones, les "sympahisants bulgares " qu'il fallait expulser de Florina étaient au nombre de 31 000. Si l'on compare ces chiffres avec le pourcentage de slavophones dans les nomes de Florina et de Kastoria, il s'ensuit que 40 à $50 \%$ des slavophones étaient prêts (ou étaient considérés comme tel par le gouvernement grec et par leurs chefs slavomacédoniens) à soutenir un soulèvement contre l'État grec ${ }^{44}$.

L'estimation de Philippe Dragoumis, député depuis des années de la région était en revanche fort différente : il pensait en effet que seul un petit nombre de slavophones appartenait aux pro-grecs (grécomans) ou aux sympathisants bulgares, alors qu'à la troisième catégorie à laquelle "appartient la grande masse des bulgarophones qui n'ont pas de conscience nationale développée mais veulent la tranquillité, la liberté et la sécurité que leur avait procuré l'État grec, est entrânée par le cours des choses ${ }^{45}$.

Combien ont été les slavophones qui, comme Slavomacédoniens, ont combattu dans les rangs de l'A.D? selon le politologue Nikos Marantzidis, qui s'appuie sur des sources yougoslaves et des sources du QG de l'armée grecque, les slavophones composaient environ 20-25\% de la force totale de l'A.D, soit environ 15-20 000 sur toute la durée de la guerre. Au contraire, l'historien de la République de Macédoine, Risto Kyriazovski, soutient que plus de 20000 «Slavomacédoniens » ont combattu dans l'A.D, comptant parmi eux les services de l'arrière.

Selon les états du NOF, en mai 1947, il avait 16349 membres inscrits, ce qui montre qu'au début de la guerre civile, il y avait un nombre relativement important de slavophones qui s'étaient enrôlées aux côtés de l'A.D ${ }^{46}$. Il est certain qu'on ne peut donner avec certitude le chiffre exact des slavophones qui ont combattu comme 
andartes dans l'A.D, ni le nombre exact de ceux qui appartenaient à ses services annexes, puisque, comme déjà dit, une grande partie de la population slavophone restée dans les villages soutenait l'A.D. On doit cependant remarquer que, les historiens de la République de Macédoine, dans leurs efforts pour présenter la guerre civile grecque comme une guerre de libération ethnique «macédonienne», citent des chiffres plutôt exagérés: ainsi un tiers des andartès de l'A.D aurait été des "Slavomacédoniens ", et dans une autre estimation excessive, les slavophones iraient jusqu'à former la majorité de l'armée de gauche dans la guerre civile ${ }^{47}$. Bien sûr, très logiquement, sur la fin de la guerre civile, en raison de la concentration des affrontements dans les régions habitées par des slavophones, leur pourcentage dans l'A.D a augmenté.

Selon des sources du KKE, les réfugiés politiques dans les pays socialistes (sauf la Yougoslavie) étaient au nombre de 55881 en 1950. Parmi eux, 20000 étaient slavophones, c'est-à-dire un pourcentage de $35 \%$ de l'ensemble des réfugiés politiques $^{48}$. En revanche, des sources de la République de Macédoine augmentent ce chiffre des Slavomacédoniens qui ont abandonné la Macédoine grecque dans la décennie 1940 à 60000 personnes et le nombre des victimes à $20000^{49}$. Plus sûre paraît l'estimation selon laquelle, dans le paidomazoma $»^{50}$ des andartès, 11000 enfants étaient slavophones, 7000 d'entre eux accompagnés de leurs parents qui s'installèrent en Yougoslavie ou en Bulgarie ${ }^{51}$.

Les données des recensements de la population sont très utiles pour suivre les variations de population dans le nome de Florina, en particulier si nous les comparons aux pourcentages de participation aux élections de 1946.

Variation de population des villages slavophones du nome de Florina entre 1940 et 1951

\begin{tabular}{|l|l|l|l|l|l|}
\hline Villages & $\begin{array}{l}\text { Population } \\
1940\end{array}$ & $\begin{array}{l}\% \\
\text { votants/ } \\
\text { pop. }\end{array}$ & $\begin{array}{l}\text { Population } \\
1951\end{array}$ & $\begin{array}{l}\text { Baisse } \\
\text { pop. }\end{array}$ & $\begin{array}{l}\% \\
\text { baisse }\end{array}$ \\
\hline Véis & 2450 & $2,69 \%$ & 2062 & 388 & $15,84 \%$ \\
\hline Achladas, Skopos & 2388 & $7,87 \%$ & 362 & 2026 & $84,84 \%$ \\
\hline Polypotamos & 2601 & $1,19 \%$ & 1777 & 824 & $31,68 \%$ \\
\hline Atrapos, Triandaphyllia & 1989 & $1,66 \%$ & 766 & 1223 & $61,49 \%$ \\
\hline Boufios & 1747 & $14,37 \%$ & 1498 & 249 & $14,25 \%$ \\
\hline Aghiou Pandeleimon & 1189 & $5,63 \%$ & 1056 & 133 & $11,19 \%$ \\
\hline Aetos & 1955 & $2,51 \%$ & 2608 & -653 & $-33,40 \%$ \\
\hline Xyno Nero & 2232 & $16,22 \%$ & 466 & 1766 & $79,12 \%$ \\
\hline $\begin{array}{l}\text { Pyxo,Agh. } \\
\text { Achillios }\end{array}$ & & & & & \\
\hline
\end{tabular}




\begin{tabular}{|l|l|l|l|l|l|}
\hline $\begin{array}{l}\text { Andartiko, Trigono, Sfika, } \\
\text { Tyrnovo }\end{array}$ & 2497 & $6,77 \%$ & 1231 & 1266 & $50,70 \%$ \\
\hline Vatochorio, Krystallopigi, Kottas & 1920 & $2,66 \%$ & 450 & 1470 & $76,56 \%$ \\
\hline Kelli & 1577 & $15,09 \%$ & 1336 & 241 & $15,28 \%$ \\
\hline Sitaria, Paliastra, Lofoi & 1913 & $12,44 \%$ & 1957 & -44 & $-2,30 \%$ \\
\hline Kratero, Ethniko, Parori & 1781 & $14,37 \%$ & 1551 & 230 & $12,91 \%$ \\
\hline Ensemble de ces villages & 26239 & $7,62 \%$ & 17120 & 9119 & $34,75 \%$ \\
\hline Ensemble du nome & 87931 & $10,11 \%$ & 69391 & 18540 & $21,08 \%$ \\
\hline
\end{tabular}

47 Du tableau ci-dessus il ressort qu'à quelques exceptions près, que plus grand a été le pourcentage des votants aux élections, plus petite a été la baisse de population dans les villages. Les slavophones qui ont voté aux élections de 1946 ont eu plus de probabilités de survie que ceux qui n'avaient pas voté, car volontairement ou non, ils ont eu la chance de s'intégrer au parti des vainqueurs. Quant aux variations de population, nous observons d'assez grandes différences d'un village à un autre, tandis qu'en moyenne la diminution dans les villages slavophones a été de près de $35 \%$. Il s'agit d'une diminution en chiffres absolus, sans tenir compte de l'augmentation normalement attendue sur une période de 11 ans. Il faut aussi signaler que ce tableau ne comprend que des villages slavophones, alors dans les villages mixtes, la polarisation a été plus grande, et donc l'enrôlement des slavophones dans l'A.D et l'exil qui s'ensuivit.

La diminution de la population slavophone dans le nome de Kastoria est encore plus impressionnante, et la comparaison avec d'autres groupes ethniques prouve l'intense polarisation de la région.

3. Population des villages de la région de Kastoria, par groupe ethnique

\begin{tabular}{|l|l|l|l|l|}
\hline Année & 1940 & 1951 & Changement & $\begin{array}{l}\% \text { change } \\
\text { ment }\end{array}$ \\
\hline Villages mixtes & 26585 & 23278 & -3307 & $-12,44 \%$ \\
\hline Slavophones & 18871 & 9889 & -8982 & $-47,60 \%$ \\
\hline “Grecs"52 & 22111 & 18318 & -3793 & $-17,15 \%$ \\
\hline Réfugiés & 4588 & 3722 & -866 & $-18,88 \%$ \\
\hline Total & 72155 & 55207 & -16948 & $-23,49 \%$ \\
\hline
\end{tabular}




\section{Conclusion}

Après la guerre civile le gouvernement grec a pris ses distances par rapport à sa politique de "purification ethnique" dont on a montré, récemment, qu'elle avait dominé les deux années qui suivirent l'Accord de Varkiza. À la fin de la guerre civile, le contexte international et la nécessité de reconnaître une minorité slave en Grèce, a conduit l'État grec a une politique qui se rapprochait davantage des propositions de Ion Dragoumis. On a considéré que les slavophones qui désertaient les rangs de l'A.D à la fin de la guerre civile avaient été enrôlés de force ou arrachés à leur village et ainsi on les autorisa à y retourner. De plus, on a même interdit aux organismes officiels de les traiter de «Bulgares », au profit désormais du nom de « Grecs slavophones ». Les agents de l'État en régions slavophones se sont vus ordonner d'aider la population à prendre confiance dans l'État, à croire qu'il pouvait la protéger et contribuer à résoudre ses problèmes $^{53}$. Les slavophones qui avaient quitté la Grèce, perdirent leur nationalité et leurs biens fonciers qui passèrent à leurs familles, à des réfugiés nationalement bienpensants ou à des autochtones qui ont pu ainsi obtenir une propriété agricole de taille vivable.

D’après les éléments statistiques de Kastoria et de Florina, on peut voir que presque la moitié des slavophones ont réussi à survivre et à rester dans leur pays. La plupart appartenaient à ceux que Keramitsis décrivait comme "pacifistes sans raison " qui " craignaient pour leur famille " et qui, en valorisant les réseaux sociaux d'avantguerre, ont réussi à rester et à s'intégrer progressivement dans la société grecque ${ }^{54}$.

51 Même s'il est impossible de définir avec précision le pourcentage des slavophones qui ont choisi ou ont été contraints de combattre aux côtés de l'A.D, il est certain que la guerre civile grecque les a divisés. Une première estimation, sur la base des données disponibles, montre que plus de $50 \%$ d'entre eux (pourcentage sensiblement plus grand dans le nome de Kastoria) a combattu aux côtés de l'A.D. Il est également impossible de préciser combien ont lutté pour l'idéologie politique communiste et l'idéologie nationale macédonienne et/ou ont été contraint par les circonstances de combattre pour l'A.D. Il est certain qu'une partie d'entre eux s'est radicalisée dans l'expérience de la l'Occupation et a cru à l'identité politique et nationale du Slavomacédonien que proposait le KKE, avec pour résultat de voir positivement un possible changement de frontières dans la région et la vie dans un éventuel régime communiste qui résulterait, espéraient-ils, du conflit armé. Cependant, dans la guerre civile qui commençait, la plupart des slavophones (comme me l'a dit un résistant Slavomacédonien de l'A.D de façon caractéristique) «ne voulaient pas quitter leur lit pour partir combattre dans la montagne $»^{55}$ et la participation active de nombre d'entre eux aux côtés du KKE a été d'abord le résultat du blocus économique et social qu'ils subissaient, et seulement au second rang seulement, la conséquence du respect politique et de la reconnaissance par le KKE, de la culture et leur identité « slavomacédonienne ». 


\section{BIBLIOGRAPHIE}

Alvanos, Raymond, (2005), « Conflits sociaux et comportements politiques dans la région de Kastoria, 1922-1949. » (en grec), Thèse de doctorat non publiée, Univ. Salonique, Sciences Politiques.

Alvanos, Raymond, (2003), « Conflits politiques et ethniques de l'entre-deux-guerres et guerre

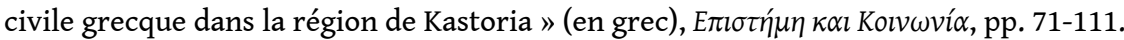

Close, David, (1998), « La reconstruction de l'État » dans Close, David, (dir.), La guerre civile grecque, 1943-1950, Études sur une polarisation, (en grec) Athènes : Filistor.

État-major général de l'Armée, Direction du Renseignement, (1986), Déposition « Fanis ", commandant A2 du bureau de la direction générale sur les Bandes, (en grec), Athènes, Imprimerie de la Gendarmerie royale, 1949, réédition, Athènes : Eleftheri Skepsi.

Kofos, Evangelos, (1989), The impact of the Macedonian question on civil conflict in Greece, 1945-1949, Athènes: ELIAMEP.

Kofos, Evangelos, (1964), Nationalism and Communism, Thessalonique, Institut d'Études Balkaniques.

Koliopoulos, Ioannis, (1995), Le pillage des pensées, la question macédonienne dans la Macédoine occidentale occupée, 1941-44, (en grec), Salonique : Vania, 2 vol.

Kostopoulos, Tasos, (2009), « La question macédonienne dans la décennie 40 », p. 363-413 dans Chatziiosif, Ch. (dir.) Histoire de la Grèce au XXe siècle, 1945-1952, Athènes : Vivliorama.

Kostopoulos, Tasos, (2000), La langue interdite (en grec), Athènes : Vivliorama.

Kyrjazovski, Risto, (1990), “The struggle of the Macedonians from Aegean Macedonia for the use of standard Macedonian", Macedonian Review, 3, vol 20.

Kyrjazovski, Risto, "The situation of the Macedonian Minority in Greece after the Second World War" Macedonian Review, 24-1-94.

Lagani, Irène, (1996), Le « Paidomazoma » et les relations gréco-yougoslaves, 1949-1953, (en grec) Athènes : I. Sidéris.

Laïou, Angeliki, (1992), « Déplacements de population dans la campagne grecque pendant la guerre civile grecque « (en grec) dans Lars Baerentzen et Ioannis Iatridis (dir.), Études sur la guerre civile 1945-1949, (en grec), Athènes : Olkos.

Michailidis, Iakovos, (2004), Les visages de Janus, les relations grécoyougoslaves à la veille de la guerre civile grecque (1944-1946) (en grec), Athènes : Patakis.

Michailidis, Iakovos, (2004), « Réfugiés politiques slavomacédoniens en Macédoine yougoslave » dans l'ouvrage collectif Réfugiés dans les Balkans. Mémoire et intégration, (en grec) Athènes : Patakis, pp. 43-82.

Sfetas, Spyridon, (2001), Vues sur la question macédonienne au XXe siècle, (en grec), Salonique : Vania. Siaperas, Kostas, (1990), Les chemins secrets de l'Armée Démocratique. De Varkiza à Bülkes, (en grec) Athènes.

Voglis, Polyméris, (2009), «La société rurale pendant la guerre civile », dans Ch. CHATZIIOSIF, Histoire de la Grèce au XXe siècle, Athènes : Vivliorama.

Zafiropoulos, Dimitrios, (1948), Le KKE et la Macédoine, (en grec), Athènes. 


\section{NOTES}

1. Dans ce texte, le mot "slavophone » a été adopté en pleine conscience qu'à l'époque de la guerre civile, la plupart des slavophones étaient bilingues et communiquaient aussi en grec. Ce terme a cependant été préféré au mot "Slavomacédoniens » qu'emploient d'autres chercheurs, car ce dernier est davantage lié à l'identité « slavomacédonienne » adoptée et promue par le KKE, particulièrement à l'époque de la guerre civile, alors que, comme on le montrera dans cet article, seule une partie des « slavophones » («Slavomacédoniens » pour le KKE ou « Macédoniens » pour le FYROM) s'identifiait à l'identité nationale slavomacédonienne ou/et à l'idéologie politique communiste.

2. $\mathrm{KKE}=$ Parti communiste grec (en grec).

3. Volontaires grecs des bandes luttant au début des années 1900 pour l'intégration de la Macédoine, encore ottomane, à la Grèce. NDT.

4. Pour les minuscules ou majuscules à Slavophones, j'ai respecté le texte original. NDT.

5. « Défense » : organisation de Slavophones « bulgarisants » à partir de 1943. NDT.

6. Pour d'autres précisions sur la période de l'entre-deux-guerres et de l'Occupation, voir Raymond Alvanos, "Conflits sociaux et comportements politiques dans la région de Kastoria, 1922-1949. » (en grec), Thèse de doctorat non publiée, Université de Salonique, Sciences Politiques, 2005.

7. 2416 familles possédaient entre 2,5 et 5 hectares, et 1417 entre 5 et 10 hectares. Enfin seules 250 familles possédaient plus de 10 hectares. AFD 92.1/32. Tableaux sur la contribution à l'étude de l'agriculture et élevage de la région de Florina, de l'agronome de l'ATE, Kavounis, avril 1945.

8. $25 \%$ de la population du département possédait une surface entre 3 et 10 hectares, seuls $0,1 \%$ avaient plus de 10 hectares. La Voix de Kastoria 7/10/45.

9. UNRRA = United Nation Relief and Rehabilitation Administration. Sur le fait que les aides de l'UNRRA, en Grèce, ont été en général dirigées de préférence vers les "nationalistes » voir

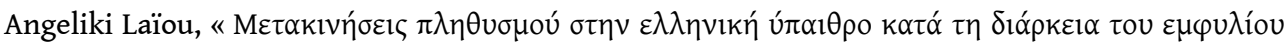

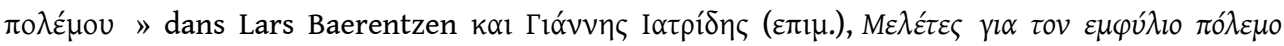
1945-1949, Athènes, Olkos, 1992, p. 75-76.

10. Iakovos Michailidis, Les visages de Janus, les relations grécoyougoslaves à la veille de la guerre civile grecque (1944-1946), en grec, Athènes, Patakis, 2004, p. 259.

11. Risto Kyrjazovski, "The situation of the Macedonian Minority in Greece after the Second World War" Macedonian Review, 24-1-94, pp.53-54, et Risto Kyrjazovski, "The struggle of the Macedonian's from Aegean Macedonia for the use of standard Macedonian", Macedonian Review, 1990, 3, vol 20, p. 192.

12. Ioannis Koliopoulos, Le pillage des pensées, la question macédonienne dans la Macédoine occidentale occupée, 1941-1944, en grec, Salonique, Vania, 1995, t 2, pp. 32-33.

13. AFD,104,4/98, lettre de Ioannis Zografos de Yannitsa à Philippe Dragoumis, 26/5/1945.

14. Kofos, Nationalism and Communism... op.cit., p. 148, Iakovos Michailidis, «Réfugiés politiques slavomacédoniens en Macédoine yougoslave» dans l'ouvrage collectif Réfugiés dans les Balkans. Mémoire et intégration, en grec, Athènes, Patakis, 2004, pp. 43-82.

15. Giannoulis, originaire d'un village du Grammos, diplômé en droit, instructeur à l'École des Officiers de Réserve de Syros, a servi dans l'ELAS de février 1943 à février 1945. Après Varkiza, il dut fuir en Yougoslavie, d'où... il revint pour devenir l'un des grands militaires de l'Armée Démocratique.

16. Dimitrios Zafiropoulos, Le KKE et la Macédoine, en grec, Athènes, 1948, p. 129.

17. Ilias Nikolakopoulos, op.cit, passim...

18. НПА : Mouvement uni des nationalistes, ЕПЕ : Union Politique Nationale, КФ : Parti Libéral.

Les chiffres concernant la population viennent du Service National Statistique de Grèce, ceux qui concernent les circonscriptions figurent dans l'annonce des élections où, en détails, outre les 
villages concernés, on trouve les alliances, les emblèmes et les candidats des partis. L'annonce en date du 26/3/1946 est signée par le préfet de Florina, AFD, 70.1/28.

19. HПЕ : Mouvement uni des nationalistes

ЕПЕ : Union Politique Nationale

КФ : Parti Libéral

Les chiffres concernant la population viennent du Service National Statistique de Grèce, ceux qui concernent les circonscriptions figurent dans l'annonce des élections où, en détails, outre les villages concernés, on trouve les alliances, les emblèmes et les candidats des partis. L'annonce en date du 26/3/1946 est signée par le préfet de Florina, AFD, 70.1/28.

20. Les tableaux pour la région de Kastoria résultent du croisement des résultats officiels des élections de 1946 (Statistique des Élections Législatives du 31 mars 1946) et d'un recensement informel des services du Ministère des Affaires Étrangères, - Tableau des Communautés et des Hameaux de la Nomarchie de Kastoria, 1945, AFD 49.6/447. J'ai préféré cette statistique au recensement de 1940, car elle était plus proche de la réalité démographique de l'époque, en montrant de façon évidente la diminution de la population de la région en raison de l'Occupation. Les données sur les circonscriptions électorales et les villages qui s'y trouvent, viennent de la proclamation des élections, AFD 70.1/28.

21. Qui recommandait l'abstention. NDT.

22. Voir Kostas Siaperas, Les chemins secrets de l'Armée Démocratique. De Varkiza à Bülkes, (en grec) Athènes, 1990, p. 43-44, et Koliopoulos, op.cit., tome 2, p. 169.

23. Evangelos Kofos, The impact of the Macedonian question on civil conflict in Greece, 1945-1949, ELIAMEP, Athènes, 1989, p.18, Iakovos Michailidis, Les visages de Janus, (en grec) op.cit., pp. 166-167 et Spyridon Sfetas, Vues sur la question macédonienne au XXe siècle, (en grec), Vania, Salonique, 2001, pp. 160-162.

24. Egejska Makedonija vo NOB, 1945, t. 2, 1975, pp. 97-106 et Egejska Makedonija vo NOB 1946, t. 3, Skopje, 1976, pp. 97-106.

25. Egejska Makedonija vo NOB, 1946, op.cit., pp 37-38, proclamation du NOD d'Edessa le 15.1.46; on y rapporte que le NOF recherche une alliance avec participation de l'EAM/KKE et Egejska Makedonija vo NOB, 1946, op.cit., 1976, p. 80-81, proclamation du NOF de Kastoria, 1946.

26. Egejska Makedonija, op.cit., p 86-93, exposé du NOF sur la situation politique de la « Macédoine de l'Égée », où l'on rapporte avoir recruté avec succès dans le village de réfugiés de Koromilia et dans le village «mixte» de Kalochori. Le but de l'organisation est, insiste-t-on, l'unité entre «Macédoniens » et réfugiés.

27. Egejska Makedonija vo NOB, 1946, op.cit., p. 37-38, proclamation NOF Edessa, 15-1-1946, où l'on rapporte que le NOF cherche un gouvernement de coalition avec la participation de l'EAM/ KKE et aussi p. 80-81, proclamation NOF de Kastoria, en janvier 1946.

28. Ibidem pp. 86-93, exposé du NOF sur la situation politique en "Macédoine de l'Égée » où l'on rapporte le succès de l'organisation auprès des réfugiés du village de Koromilia et du village mixte de Kalochori. On insiste sur le fait que le but de l'organisation est l'union entre « Macédoniens » et réfugiés.

29. Exormisi, $1^{\mathrm{er}}$ janvier 1948.

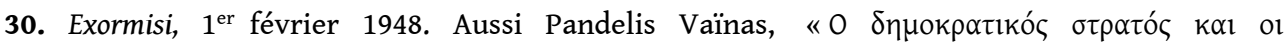

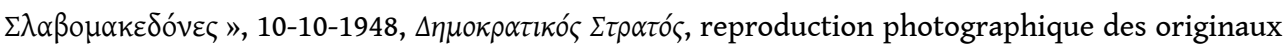
du périodique «Armée Démocratique » par le Rizospastis, t. 1, pp. 408-411, 1996.

31. MAY: Unités de Sécurité en milieu rural et MAD : détachements de poursuite = milices paramilitaires locales équipées par l'État à partir d'octobre 1946 pour traquer efficacement les hommes de gauche.

32. Bien que la supposée alliance Liakopoulos/andartes ne soit pas une réalité, il faut remarquer à quel point la mémoire des témoins l'a «sacré » collaborateur des andartès, d'autant que l'on comprit, après coup, que ses actes en définitive ne servaient que les andartès. L'argument selon 
lequel il y eut «départ forcé » vers la montagne à cause des persécutions repose sur une base réelle. Mais, bien sûr, il est logique que les témoignages à ce sujet insistent sur ce schéma explicatif qui les excuse et nie leurs responsabilités personnelles, leurs choix conscients de s'intégrer dans une armée dont nous savons maintenant qu'elle a été vaincue.

33. État-major général de l'Armée, Direction du Renseignement, Déposition " Fanis », commandant A2 du bureau de la direction générale sur les Bandes, Athènes, Imprimerie de la Gendarmerie royale, 1949, réédition, Athènes, Eleftheri Skepsi, 1986, p. 30.

34. Les guillemets sont destinés à distinguer les réfugiés de 1922 des personnes (le terme utilisé en grec est différent) qui fuient les combats ou sont chassées par l'Armée gouvernementale. NDT. 35. Voix de Kastoria, 19/19/47, 26/10/47 et 17/6/51.

36. Rapporté dans Koliopoulos, op.cit, t. 2, p. 196.

37. En juin 1947, la ligne "nettoyer le pays des slavophones ", semble avoir dominé dans la société grecque. Le député de Kastoria, lui-même réfugié, Périclès Iliadis, s'exprimant à l'Assemblée (au milieu des applaudissements enthousiastes des députés) appela l'État grec «à se débarrasser définitivement" des slavophones qui sont "le lien malhonnête qui unit le parti communiste aux Slaves de l'extérieur». Les députés saluèrent avec enthousiasme cette proposition et, même, proposèrent que son discours soit imprimé et distribué gratuitement. $L a$ Voix de Kastoria, 1 juin 1947 et 18 avril 1948.

38. AFD, 104.6/161, mémoire de 20 pages dactylographiées de Philippe Dragoumis adressé à l'état-major général d'Armée, au Roi, au secrétaire d'État aux Affaires extérieures, aux généraux Kitrilakis et Giatzi et aux préfets de Florina et Kastoria (Bonis) en date du 12 novembre 1948, avec pour titre : À propos des populations bulgarophones frontalières.

39. QG.DIS, t. 5, 60, exposé des opérations du mois d'avril 1947, XV Division au II Corps d'Armée.

40. Tasos Kostopoulos, La langue interdite (en grec), Vivliorama, 2000, pp. 206-210.

41. Giorgos Margaritis, op.cit, p. 16-18.

42. Tasos Kostopoulos, «La question macédonienne dans la décennie 40 », p. 363-413 dans Chatsiiosif

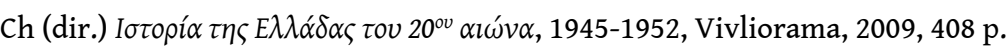

43. Egejska Makedonija vo NOB 1947, t. 6, 1980, introduction du secrétaire du NOF, Keramitsis, 20-5-1947, pp. 158-162.

44. Bibliothèque Gennadios, $A \Phi \Delta$ 104,5/134, Ministère de l'Ordre Public, $n^{\circ} 115 / 10 / 26 / 78$ au

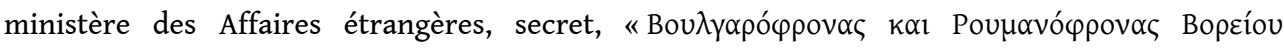

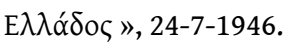

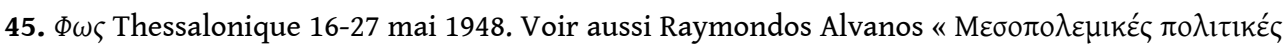

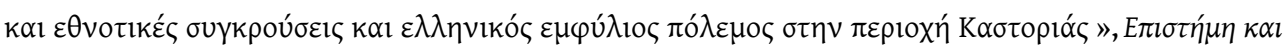

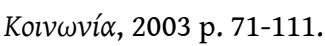

46. 6331 personnes inscrites au NOF, 4509 au AMG (Front antifasciste des femmes) et 5509 au NOMS (Union nationale de libération de la Jeunesse). 4587 inscrits dans la région de Kastoria, 4004 dans la région de Florina, 5515 dans la région d'Edessa, 1401 dans celle de GiannitsaGoumenissa et 1044 dans la région de Naoussa, Egejska Makedonija vo NOB, 1947, 1980, p. 162 exposé de Keramitziev le 20-5-1947. La présence du NOF en Macédoine orientale où les slavophones avaient peu de contacts avec l'idéologie nationale (slavo)macédonienne, était quasi

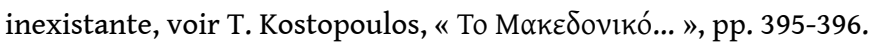

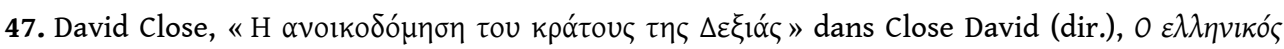

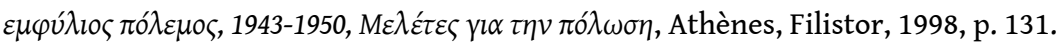

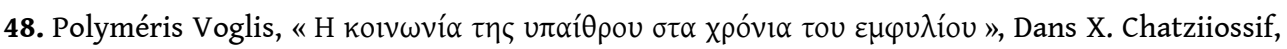

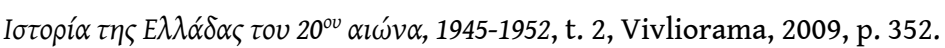

49. Tasos Kostopoulos $H \alpha \pi \alpha \gamma o \rho \varepsilon v \mu \varepsilon ́ v \eta \gamma \lambda \omega ́ \sigma \sigma \alpha$, op.cit. p. 219. 
50. Terme utilisé par les adversaires (il rappelle la levée ottomane des enfants) pour désigner l'envoi, voulu ou non par les parents, par l'A.D des enfants exposés aux combats de l'autre côté des frontières.

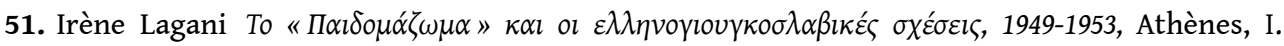
Sidéris, 1996, p. 66, utilise des données de la représentation française à l'U.N.S.C.O.B.

52. L'auteur a repris la terminologie officielle. Le fait que l'on distingue les "Grecs» (autochtones) des Réfugiés révèle l'intégration difficile de ces derniers. NDT.

53. Risto Kirjazovski, “The situation of the Macedonian...”, p. 57.

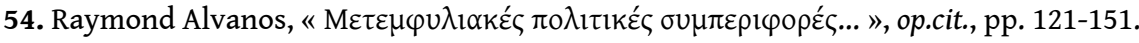

55. Manis Giorof, interview, Skopje, 12-7-2000.

\section{RÉSUMÉS}

Combien de slavophones de Macédoine grecque se sont engagés dans l'Armée Démocratique pendant la guerre civile grecque? L'ont-ils fait par communisme, par sentiment national macédonien ou contraints par les circonstances? Nul ne peut aujourd'hui fournir une réponse certaine à ces questions. L'état actuel des recherches permet cependant de fournir des approximations numériques, des précisions sur leurs conditions de vie, soumises aux pressions idéologiques des différents occupants, du gouvernement grec, du KKE, de la Yougoslavie naissante, face à une extrême pauvreté aggravée par la politique d'exclusion menée par les gouvernements grecs au départ des Allemands. On comprend également que les Slavophones sont loin d'avoir une position politique unique, contrairement à ce que qu'affirment les différentes propagandes nationales qui les entourent.

How many Slavophones from Greek Macedonia were committed in the Democratic Army during the Greek civil war? Have they joined because of communists beliefs, following their national Macedonian feeling or were they being forced by other circumstances? No one can provide a definite answer to those questions. The current state of research, however, allows some numerical approximations and details on their living conditions, revealing ideological pressures by various occupants, the Greek government, the Greek communist party, the nascent Yugoslavia, and extreme poverty, exacerbated by an exclusion policy pursued by the Greek government, after the Germans had left the country. We also understand that Slavophones are far from having a single policy position, contrary to the assertions of the various national propaganda.

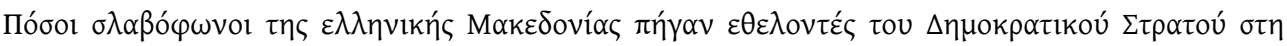

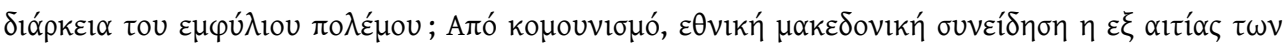

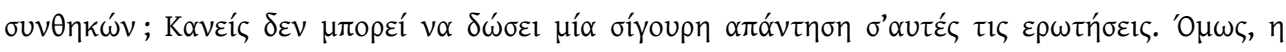

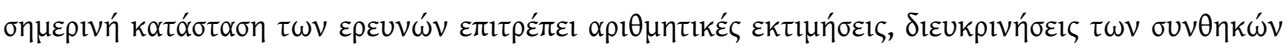

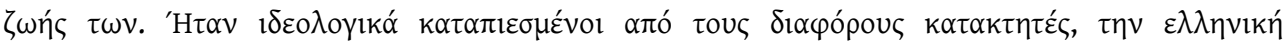

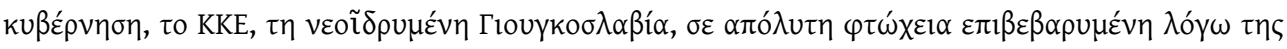

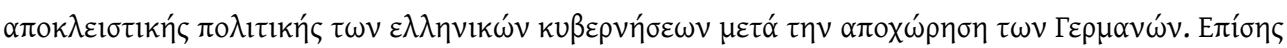

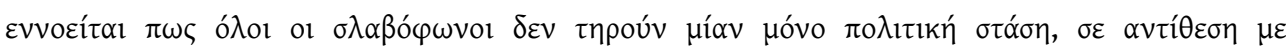

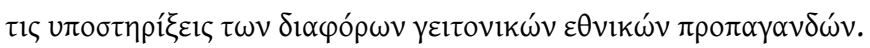


INDEX

Index chronologique : guerre civile grecque (1946-1949)

Thèmes : Histoire

motsclestr Makedonya, Ferizovik, Florina, Kastoria, Pella, Yenice Vardar, Yugoslavya,

Kaïmaktsalan, Grammos

Keywords : Greek civil war (1946-1949), Greeks slavophones, KKE, Macedonia, nation, occupation, Florina, Kastoria, Pella, Yannitsa, Yugoslavia, Skopje, Kaïmaktsalan, Grammos, History

motsclesmk ЈУГОСЛАВИЈА, ЛЕРИН, МАКЕДОНИЈА

Index géographique : Macédoine, Florina, Kastoria, Pella, Yannitsa, Yougoslavie, Skopje, Grammos, Kaïmaktsalan

Mots-clés : Armée démocratique, KKE, Comitadji, IPE, Occupation, nation, EPE, slavophones grecs, KF, KKE, EAM, ELAS, MAD, MAY, NOF, Ohrana, Slavophone, SNOF, UNRRA,

Macédonomaque, Metaxás Ioannis (1871-1941)

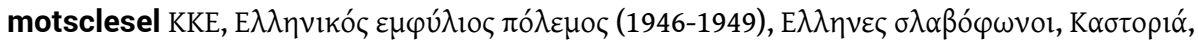

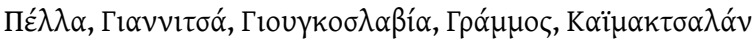

